\title{
WEIL DIFFEOLOGY I: CLASSICAL DIFFERENTIAL GEOMETRY
}

\author{
HIROKAZU NISHIMURA
}

\begin{abstract}
Topos theory is a category-theoretical axiomatization of set theory. Model categories are a category-theoretical framework for abstract homotopy theory. They are complete and cocomplete categories endowed with three classes of morphisms (called fibrations, cofibrations and weak equivalences) satisfying certain axioms. We would like to present an abstract framework for classical differential geometry as an extension of topos theory, hopefully comparable with model categories for homotopy theory. Functors from the category $\mathfrak{W}$ of Weil algebras to the category Sets of sets are called Weil spaces by Wolfgang Bertram and form the Weil topos after Eduardo J. Dubuc. The Weil topos is endowed intrinsically with the Dubuc functor, a functor from a larger category $\widetilde{\mathfrak{W}}$ of cahiers algebras to the Weil topos standing for the incarnation of each algebraic entity of $\widetilde{\mathfrak{W}}$ in the Weil topos. The Weil functor and the canonical ring object are to be defined in terms of the Dubuc functor. The principal objective of this paper is to present a category-theoretical axiomatization of the Weil topos with the Dubuc functor intended to be an adequate framework for axiomatic classical differential geometry. We will give an appropriate formulation and a rather complete proof of a generalization of the familiar and desired fact that the tangent space of a microlinear Weil space is a module over the canonical ring object.
\end{abstract}

\section{INTRODUCTION}

Differential geometry usually exploits not only the techniques of differentiation but also those of integration. In this paper we would like to use the term "differential geometry" in its literal sense, that is, genuinely differential geometry, which is vast enough as to encompass a large portion of the theory of connections and the core of the theory of Lie groups. Now we know well that there is a horribly deep and overwhelmingly gigantic valley between differential calculus of the 17th and 18th centuries (that is to say, that of the good old days of Newton, Leibniz, Lagrange, Laplace, Euler, etc.) and that of our modern age since the 19th century when Angustin Louis Cauchy was active. The former exquisitely resorts to nilpotent infinitesimals, while the latter grasps differentiation in terms of limits by using the so-called $\varepsilon-\delta$ arguments formally. Differential geometry based on the latter style of differentiation is generally called smootheology, while we propose that differential geometry based on the former style of differentiation might be called Weilology.

$M S C$ (2010): primary 58A03; secondary $18 \mathrm{~F} 99$.

Keywords: diffeology, axiomatic differential geometry, Weil algebra, Weil space, Weilology, synthetic differential geometry, topos theory, smootheology. 
As is well known, the category of topological spaces and continuous mappings is not cartesian closed. The classical example of a convenient category of topological spaces for working topologisits was suggested by Norman Steenrod [28] in the middle of the 1960s, namely, the category of compactly generated spaces. Now the category of finite-dimensional smooth manifolds and smooth mappings is not cartesian closed, either. Convenient categories for smootheology have been proposed by several authors in several corresponding forms. Among them, Souriau's [26] approach based upon the category $\mathfrak{O}$ of open subsets $O$ 's of $\mathbf{R}^{n}$ 's and smooth mappings between them has developed into a galactic volume of diffeology, for which the reader is referred to [8]. A diffeological space is a set $X$ endowed with a subset $\mathcal{D}(O) \subseteq X^{O}$ for each $O \in \mathfrak{O}$ such that, for any morphism $f: O \rightarrow O^{\prime}$ in $\mathfrak{O}$ and any $\gamma \in \mathcal{D}\left(O^{\prime}\right)$, we have $\gamma \circ f \in \mathcal{D}(O)$. A diffeological map between diffeological spaces $(X, \mathcal{D})$ and $\left(X, \mathcal{D}^{\prime}\right)$ is a mapping $f: X \rightarrow X^{\prime}$ such that, for any $O \in \mathfrak{D}$ and any $\gamma \in \mathcal{D}(O)$, we have $f \circ \gamma \in \mathcal{D}^{\prime}(O)$.

Roughly speaking, there are two approaches to geometry in representing spaces, namely, contravariant (functional) and covariant (parameterized) ones, for which the reader is referred, e.g., to Chapter 3 of [25] as well as [23] and [24]. Diffeology finds itself in the covariant realm. The contravariant approach boils down spaces to their function algebras. We are now accustomed to admitting all algebras to stand for abstract spaces in some way or other, whatever they may be. This has been a long tradition of algebraic geometry since as early as Alexander Grothendieck. Now we are ready to acknowledge any functor $\mathfrak{O}^{\mathrm{op}} \rightarrow$ Sets as an abstract diffeological space. Then, it is pleasant to enjoy

Theorem 1.1. The category of abstract diffeological spaces and natural transformations between them is a topos.

Turning to Weilology, a space should be represented by a functor Inf $^{\text {op }} \rightarrow$ Sets, where Inf stands for the category of nilpotent infinitesimal spaces. Since our creed tells us that the category $\mathbf{I n f}^{\text {op }}$ is equivalent to $\mathfrak{W}$, a space should be no other than a functor $\mathfrak{W} \rightarrow$ Sets, for which Wolfgang Bertram [6] coins the term "Weil space". To be sure, we have

Theorem 1.2. The category of Weil spaces and natural transformations between them is a topos.

\section{Cahiers algebras}

Unless stated to the contrary, our base field is assumed to be $\mathbf{R}$ (real numbers) throughout the paper so that we will often say "Weil algebra" simply in place of "Weil R-algebra". For the exact definition of a Weil algebra, the reader is referred to $\S \mathrm{I} .16$ of $[9]$.

Notation 2.1. We denote by $\mathfrak{W}$ the category of Weil algebras.

Remark 2.2. $\mathbf{R}$ is itself a Weil algebra, and it is an initial object in the category W.

Definition 2.3. An $\mathbf{R}$-algebra isomorphic to an $\mathbf{R}$-algebra of the form

$$
\mathbf{R}\left[X_{1}, \ldots, X_{n}\right] \otimes W
$$


with $\mathbf{R}\left[X_{1}, \ldots, X_{n}\right]$ being the polynomial algebra over $\mathbf{R}$ in indeterminates $X_{1}, \ldots$, $X_{n}$ (possibly $n=0$, when the definition degenerates to Weil algebras) and $W$ being a Weil algebra is called a cahiers algebra.

Remark 2.4. This definition of a cahiers algebra is reminiscent of that in the definition of Cahiers topos, where we consider a product of a Cartesian space $\mathbf{R}^{n}$ and a formal dual of a Weil algebra.

Notation 2.5. We denote by $\widetilde{\mathfrak{W}}$ the category of cahiers algebras.

Remark 2.6. The category $\mathfrak{W}$ is a full subcategory of the category $\widetilde{\mathfrak{W}}$. Both are closed under the tensor product $\otimes$.

Notation 2.7. We will use such a self-explanatory notation as $Z \rightarrow X /\left(X^{2}\right)$ or $X /\left(X^{2}\right) \leftarrow Z$ for the morphism $\mathbf{R}[Z] \rightarrow \mathbf{R}[X] /\left(X^{2}\right)$ assigning $X$ modulo $\left(X^{2}\right)$ to $Z$.

\section{WEIL SPACES}

Definition 3.1. A Weil space is simply a functor $F$ from the category $\mathfrak{W}$ of Weil algebras to the category Sets of sets. A Weil morphism from a Weil space $F$ to another Weil space $G$ is simply a natural transformation from the functor $F$ to the functor $G$.

Remark 3.2. The term "Weil space" is coined in [6].

Example 3.3. The Weil prolongation of a "manifold" in its broadest sense (cf. [4]) by a Weil algebra was fully discussed by Bertram and Souvay, for which the reader is cordially referred to [5]. We are happy to know that any manifold naturally gives rise to its associated Weil space, which can be regarded as a functor from the category of manifolds to the category Weil. It should be stressed without exaggeration that the functor is not full in general, for which the reader is referred to exuberantly readable $\S 1.6$ (discussion) of [6].

Example 3.4. The Weil prolongation $A \otimes W$ of a $C^{\infty}$-algebra $A$ by a Weil algebra $W$ was discussed in Theorem III.5.3 of [9]. We are happy to know that any $C^{\infty}$-algebra naturally gives rise to its associated Weil space.

Notation 3.5. We denote by Weil the category of Weil spaces and Weil morphisms.

Remark 3.6. Dubuc [7] has indeed proposed the topos Weil as the first step towards the well adapted model theory of synthetic differential geometry, but we would like to contend somewhat radically that the topos Weil is verbatim the central object of study in classical differential geometry

It is well known (cf. Chapter 1 of [13]) that

Theorem 3.7. The category Weil is a topos. In particular, it is locally cartesian closed.

Remark 3.8. Dubuc [7] has called the category Weil the Weil topos. 
Remark 3.9. The category of Frölicher spaces is indeed cartesian closed, but it is not locally cartesian closed. On the other hand, the category of diffeological spaces is locally cartesian closed. For these matters, the reader is referred to [27]. It was shown by Baez and Hoffnung [2] that diffeological spaces as well as Chen spaces are no other than concrete sheaves on concrete sites.

Definition 3.10. The Weil prolongation $F^{W}$ of a Weil space $F$ by a Weil algebra $W$ is simply the composition of the functor $\left(_{-}\right) \otimes W: \mathfrak{W} \rightarrow \mathfrak{W}$ and the functor $F: \mathfrak{W} \rightarrow$ Sets, namely

$$
F((-) \otimes W): \mathfrak{W} \rightarrow \text { Sets }
$$

which is surely a Weil space.

Remark 3.11. ( $\left(_{-}^{(\cdot)}\right.$ assigning $F^{W}$ to each $(W, F) \in \mathfrak{W} \times$ Weil can naturally be regarded as a bifunctor $\mathfrak{W} \times$ Weil $\rightarrow$ Weil.

Trivially, we have

Proposition 3.12. For any Weil space $F$ and any Weil algebras $W_{1}$ and $W_{2}$, we have

$$
\left(F^{W_{1}}\right)^{W_{2}}=F^{W_{1} \otimes W_{2}} .
$$

Remark 3.13. The so-called Yoneda embedding

$$
\mathbf{y}: \mathfrak{W}^{\mathrm{op}} \rightarrow \text { Weil }
$$

is full and faithful. The famous Yoneda lemma claims that

$$
F\left({ }_{-}\right) \cong \operatorname{Hom}_{\text {Weil }}\left(\mathbf{y}\left({ }_{-}\right), F\right)
$$

for any Weil space $F$. The Yoneda embedding can be extended to

$$
\widetilde{\mathbf{y}}: \widetilde{\mathfrak{W}}^{\mathrm{op}} \rightarrow \text { Weil }
$$

by

$$
\widetilde{\mathbf{y}}(A)=\operatorname{Hom}_{\mathbf{R}-\mathbf{A l g}}\left(A,{ }_{-}\right)
$$

for any $A \in \widetilde{\mathfrak{W}}$, where $\mathbf{R}-\mathbf{A l g}$ denotes the category of $\mathbf{R}$-algebras.

Remark 3.14. Given Weil algebras $W_{1}$ and $W_{2}$, we have

$$
\mathbf{y} W_{1} \times \mathbf{y} W_{2} \cong \mathbf{y}\left(W_{1} \otimes W_{2}\right) .
$$

Remark 3.15. As is well known (cf. $\S 8.7$ of [1]), given Weil spaces $F$ and $G$, their exponential $F^{G}$ in Weil is provided by

$$
\operatorname{Hom}_{\text {Weil }}\left(\mathbf{y}_{-} \times G, F\right) \text {. }
$$

Proposition 3.16. For any Weil space $F$ and any Weil algebra $W, F^{W}$ and $F^{\mathbf{y} W}$ are naturally isomorphic, namely,

$$
F^{W} \cong F^{\mathbf{y} W}
$$

where the left-hand side stands for the Weil prolongation $F^{W}$ of $F$ by $W$, while the right-hand side stands for the exponential $F^{\mathbf{y} W}$ in the topos Weil. 
Proof. The proof is so simple as follows:

$$
\begin{aligned}
& F^{\mathbf{y} W}=\operatorname{Hom}\left(\mathbf{y}_{-} \times \mathbf{y} W, F\right) \\
& {[(3.3)]} \\
& \cong \operatorname{Hom}(\mathbf{y}(-\otimes W), F) \\
& {[(3.2)]} \\
& \cong F(-\otimes W) \\
& {[(3.1)]} \\
& \quad=F^{W} .
\end{aligned}
$$

Corollary 3.17. Given a Weil algebra $W$ together with Weil spaces $F$ and $G$, $\left(F^{G}\right)^{W}$ and $\left(F^{W}\right)^{G}$ are naturally isomorphic, namely,

$$
\left(F^{G}\right)^{W} \cong\left(F^{W}\right)^{G}
$$

Proof. We have

$$
\begin{aligned}
\left(F^{G}\right)^{W} & \cong\left(F^{G}\right)^{\mathbf{y} W} \\
& {[\text { by Proposition 3.16] }} \\
& \cong\left(F^{\mathbf{y} W}\right)^{G} \\
& \cong\left(F^{W}\right)^{G} \\
& {[\text { by Proposition 3.16]. }}
\end{aligned}
$$

Corollary 3.18. For any Weil algebra $W$, the functor $\left({ }_{-}\right)^{W}$ : Weil $\rightarrow$ Weil preserves limits, particularly, products.

Proof. Since the functor $\left({ }_{-}\right)^{W}$ is of its left adjoint $\left({ }_{-}\right) \times \mathbf{y} W$ (cf. Proposition 8.13 of [1]), the desired result follows readily from the well known theorem claiming that a functor, being of its left adjoint, preserves limits (cf. Proposition 9.14 of [1]).

Notation 3.19. We denote by $\mathbb{R}$ the forgetful functor $\mathfrak{W} \rightarrow$ Sets, which is surely a Weil space. It can also be defined as

$$
\mathbb{R}=\widetilde{\mathbf{y}}(\mathbf{R}[X]) .
$$

Remark 3.20. The Weil space $\mathbb{R}$ is canonically regarded as an $\mathbf{R}$-algebra object in the category Weil.

Remark 3.21. Since $\mathbb{R}$ is an $\mathbf{R}$-algebra object in the category Weil, we can define, after $\S I .16$ of [9], another $\mathbf{R}$-algebra object $\mathbb{R} \otimes W$ in the category Weil for any Weil algebra $W$.

Notation 3.22. We denote by $\mathbf{R}-\mathbf{A l g}$ (Weil) the category of $\mathbf{R}$-algebra objects in the category Weil. 
Proposition 3.23. The functors

$$
\mathbb{R}^{\mathbf{y}(-)}, \mathbb{R} \otimes\left({ }_{-}\right): \mathfrak{W} \rightarrow \mathbf{R}-\mathbf{A l g}(\text { Weil })
$$

are naturally isomorphic.

Proof. We have

$$
\begin{aligned}
\mathbb{R}^{\mathbf{y} W}\left(W^{\prime}\right) & \cong \mathbb{R}^{W}\left(W^{\prime}\right) \\
& {[\text { By Proposition 3.16] }} \\
& =W^{\prime} \otimes W
\end{aligned}
$$

\section{Microlinearity}

Not all Weil spaces are susceptible to the techniques of classical differential geometry so that there should be a criterion by which we can select the decent ones.

Definition 4.1. A Weil space $F$ is called microlinear provided that a finite limit diagram $\mathcal{D}$ in $\mathfrak{W}$ always yields a limit diagram $F^{\mathcal{D}}$ in Weil.

Proposition 4.2. We have the following:

(1) The Weil space $\mathbb{R}$ is microlinear.

(2) The limit of a diagram of microlinear Weil spaces is microlinear.

(3) Given Weil spaces $F$ and $G$, if $F$ is microlinear, then the exponential $F^{G}$ is also microlinear.

Proof. The first statement follows from Proposition 3.23. The second statement follows from the well-known fact that double limits commute. The third statement follows from Corollary 3.17.

It is easy to see that

Proposition 4.3. A Weil space $F$ is microlinear iff the diagram

$$
F(W \otimes \mathcal{D})
$$

is a limit diagram for any Weil algebra $W$ and any finite limit diagram $\mathcal{D}$ of Weil algebras.

Proof. By Proposition 8.7 of [1].

\section{Weil CATEgories}

Definition 5.1. A Weil category is a pair $(\mathcal{K}, \mathbf{D})$, where

(1) $\mathcal{K}$ is a topos.

(2) $\mathbf{D}: \widetilde{\mathfrak{W}}^{\mathrm{op}} \rightarrow \mathcal{K}$ is a product-preserving functor. In particular, we have

$$
\mathbf{D}(\mathbf{R})=1
$$

where 1 denotes the terminal object in $\mathcal{K}$.

Remark 5.2. The entity $\mathbf{D}$ is called a Dubuc functor enthroning his pioneering work in [7]. 
Now some examples are in order.

Example 5.3. The first example of a Weil category has already been discussed in $\S 3$, namely,

$$
\begin{aligned}
& \mathcal{K}=\text { Weil, } \\
& \mathbf{D}=\tilde{\mathbf{y}} .
\end{aligned}
$$

Indeed, this is the paradigm of our new concept of a Weil category, just as the category Sets is the paradigm of the prevailing concept of a topos.

Notation 5.4. We denote by $C^{\infty}-\mathbf{A l g}$ the category of $C^{\infty}$-algebras.

Example 5.5. Let $\mathbf{L}$ be a class of $C^{\infty}$-algebras encompassing all $C^{\infty}$-algebras of the form $C^{\infty}\left(\mathbf{R}^{n}\right) \otimes W$ with $W$ being a Weil algebra (cf. Theorem III.5.3 of [9].). We define a functor $i_{\widetilde{\mathfrak{W}}, C^{\infty}-\mathrm{Alg}}: \widetilde{\mathfrak{W}} \rightarrow C^{\infty}-\mathbf{A l g}$ as

$$
i_{\widetilde{\mathfrak{W}}, C^{\infty}-\mathbf{A l g}}\left(\mathbf{R}\left[X_{1}, \ldots, X_{n}\right] \otimes W\right)=C^{\infty}\left(\mathbf{R}^{n}\right) \otimes W .
$$

Putting down $\mathbf{L}$ as a full subcategory of the category $C^{\infty}-\mathbf{A l g}$, consider a subcanonical Grothendieck topology $J$ on the category $\mathbf{L}^{\text {op }}$. We let $\mathcal{K}$ be the category of all sheaves on the site $\left(\mathbf{L}^{\mathrm{op}}, J\right)$. The Dubuc functor $\mathbf{D}$ is defined as

$$
\mathbf{D}=\mathbf{y} \circ i_{\widetilde{\mathfrak{W}}, C^{\infty}-\mathbf{A l g}}
$$

where $\mathbf{y}$ stands for the Yoneda embedding.

Remark 5.6. Such examples have been discussed amply in the context of welladapted models of synthetic differential geometry being not aware at all of Weil categories. The reader is referred to [9] and [14] for them.

Now we fix a Weil category $(\mathcal{K}, \mathbf{D})$ throughout the rest of this section. Weil functors are to be defined within our framework of a Weil category.

Definition 5.7. The bifunctor $\mathbf{T}: \mathfrak{W} \times \mathcal{K} \rightarrow \mathcal{K}$ is defined to be

$$
\mathbf{T}((-),(\cdot)) \cong(\cdot)^{\mathbf{D}(-)} .
$$

We give some elementary properties with respect to $\mathbf{T}$.

Proposition 5.8. We have the following:

(i) The functor $\mathbf{T}(\mathbf{R},(-))$ and the identity functor of $\mathcal{K}$, both of which are $\mathcal{K} \rightarrow \mathcal{K}$, are naturally isomorphic, namely,

$$
\mathbf{T}\left(\mathbf{R},\left(_{-}\right)\right) \cong\left({ }_{-}\right) .
$$

(ii) The trifunctors $\mathbf{T}\left(\left(\cdot \cdot_{2}\right), \mathbf{T}\left(\left(\cdot_{1}\right),(-)\right)\right)$ and $\mathbf{T}\left(\left(\cdot \cdot_{1}\right) \otimes\left(\cdot_{2}\right),(-)\right)$, both of which are $\mathfrak{W} \times \mathfrak{W} \times \mathcal{K} \rightarrow \mathcal{K}$, are naturally isomorphic, namely,

$$
\mathbf{T}\left(\left(\cdot_{2}\right), \mathbf{T}\left(\left(\cdot \cdot_{1}\right),(-)\right)\right) \cong \mathbf{T}\left(\left(\cdot \cdot_{1}\right) \otimes\left(\cdot_{2}\right),\left({ }_{-}\right)\right)
$$

for any Weil space $F$ and any Weil algebras $W_{1}$ and $W_{2}$.

Proposition 5.9. Given a Weil algebra $W$, the functor $\mathbf{T}(W, \cdot): \mathcal{K} \rightarrow \mathcal{K}$ preserves limits.

Proof. Since the functor $\mathbf{T}(W, \cdot): \mathcal{K} \rightarrow \mathcal{K}$ is of its left adjoint $(\cdot) \times \mathbf{D} W: \mathcal{K} \rightarrow$ $\mathcal{K}$, the desired result follows readily from the well-known theorem claiming that a functor being of its left adjoint preserves limits (cf. Proposition 9.14 of [1]). 
Proposition 5.10. The trifunctors $\mathbf{T}\left((-),\left(\cdot_{1}\right)^{(\cdot 2)}\right), \mathbf{T}\left((-),\left(\cdot{ }^{1}\right)\right)^{(\cdot 2)}: \mathfrak{W} \times \mathcal{K} \times$ $\mathcal{K} \rightarrow \mathcal{K}$ are naturally isomorphic, namely,

$$
\mathbf{T}\left((-),(\cdot 1)^{(\cdot 2)}\right) \cong \mathbf{T}((-),(\cdot 1))^{(\cdot 2)} .
$$

Proof. We have

$$
\begin{aligned}
\mathbf{T}\left(\left({ }_{-}\right),\left(\cdot_{1}\right)^{\left(\cdot \cdot_{2}\right)}\right) & =\left(\left(\cdot_{1}\right)^{\left(\cdot \cdot_{2}\right)}\right)^{\mathbf{D}(-)} \\
& \cong\left(\left(\cdot_{1}\right)^{\mathbf{D}(-)}\right)^{\left(\cdot \cdot_{2}\right)} \\
& \left.=\mathbf{T}\left((-),(\cdot)_{1}\right)\right)^{\left(\cdot \cdot_{2}\right)}
\end{aligned}
$$

An $\mathbf{R}$-algebra object is to be introduced within our framework of a Weil category.

Notation 5.11. The entity $\mathbf{D}(\mathbf{R}[X])$ is denoted by $\mathbb{R}$.

It is in nearly every mathematician's palm to see that

Proposition 5.12. The entity $\mathbb{R}$ is a commutative $\mathbf{R}$-algebra object in $\mathcal{K}$ with respect to the following addition, multiplication, scalar multiplication by $\alpha \in \mathbf{R}$ and unity:

$$
\begin{gathered}
\mathbf{D}(X+Y \leftarrow X): \mathbb{R} \times \mathbb{R}=\mathbf{D}(\mathbf{R}[X, Y]) \rightarrow \mathbf{D}(\mathbf{R}[X])=\mathbb{R}, \\
\mathbf{D}(X Y \leftarrow X): \mathbb{R} \times \mathbb{R}=\mathbf{D}(\mathbf{R}[X, Y]) \rightarrow \mathbf{D}(\mathbf{R}[X])=\mathbb{R}, \\
\mathbf{D}(\alpha X \leftarrow X): \mathbb{R}=\mathbf{D}(\mathbf{R}[X]) \rightarrow \mathbf{D}(\mathbf{R}[X])=\mathbb{R}, \\
\mathbf{D}(1 \leftarrow X): 1=\mathbf{D}(\mathbf{R}) \rightarrow \mathbf{D}(\mathbf{R}[X])=\mathbb{R} .
\end{gathered}
$$

Notation 5.13. The above four morphisms are denoted by

$$
\begin{aligned}
+_{\mathbb{R}} & : \mathbb{R} \times \mathbb{R} \rightarrow \mathbb{R}, \\
\cdot_{\mathbb{R}} & : \mathbb{R} \times \mathbb{R} \rightarrow \mathbb{R}, \\
\alpha \cdot & : \mathbb{R} \rightarrow \mathbb{R}, \\
1_{\mathbb{R}} & : 1 \rightarrow \mathbb{R} .
\end{aligned}
$$

in order.

Notation 5.14. The entity $\mathbf{D}\left(\mathbf{R}[X] /\left(X^{2}\right)\right)$ is denoted by $D$.

Proposition 5.15. The $\mathbf{R}$-algebra object $\mathbb{R}$ operates canonically on $D$ in $\mathcal{K}$. To be specific, we have the following morphism:

$$
\begin{gathered}
\mathbf{D}\left(Z X /\left(X^{2}\right) \leftarrow X /\left(X^{2}\right)\right): \mathbb{R} \times D=\mathbf{D}(\mathbf{R}[Z]) \times \mathbf{D}\left(\mathbf{R}[X] /\left(X^{2}\right)\right)= \\
\mathbf{D}\left(\mathbf{R}[X, Z] /\left(X^{2}\right)\right) \rightarrow \mathbf{D}\left(\mathbf{R}[X] /\left(X^{2}\right)\right)=D .
\end{gathered}
$$

Notation 5.16. The above morphism is denoted by $\cdot \mathbb{R}, D$.

Proposition 5.17. It makes the following diagrams commutative: 
(1)

$$
\begin{array}{rll}
\mathbb{R} \times \mathbb{R} \times D & \longrightarrow & \mathbb{R} \times D \\
& \searrow & \downarrow \\
& D
\end{array}
$$

where the horizontal arrow is $+_{\mathbb{R}} \times D: \mathbb{R} \times \mathbb{R} \times D \rightarrow \mathbb{R} \times D$, the vertical arrow is $\cdot \mathbb{R}, D: \mathbb{R} \times D \rightarrow D$, and the slant arrow is

$$
\begin{aligned}
& \mathbf{D}\left(Z_{1} X+Z_{2} X /\left(X^{2}\right) \leftarrow X /\left(X^{2}\right)\right): \mathbb{R} \times \mathbb{R} \times D \\
= & \mathbf{D}\left(\mathbf{R}\left[Z_{1}\right]\right) \times \mathbf{D}\left(\mathbf{R}\left[Z_{2}\right]\right) \times \mathbf{D}\left(\mathbf{R}[X] /\left(X^{2}\right)\right) \\
= & \mathbf{D}\left(\mathbf{R}\left[Z_{1}, Z_{2}, X\right] /\left(X^{2}\right)\right) \rightarrow \mathbf{D}\left(\mathbf{R}[X] /\left(X^{2}\right)\right)=D .
\end{aligned}
$$

(2)

$$
\begin{array}{lll}
\mathbb{R} \times \mathbb{R} \times D & \longrightarrow & \mathbb{R} \times D \\
\downarrow & \downarrow \\
\mathbb{R} \times D & \longrightarrow & D
\end{array}
$$

where the upper horizontal arrow is $\cdot \mathbb{R} \times D: \mathbb{R} \times \mathbb{R} \times D \rightarrow \mathbb{R} \times D$, the lower horizontal arrow is $\cdot_{\mathbb{R}, D}:, \mathbb{R} \times D \rightarrow D$ the left vertical arrow is $\mathbb{R} \times \cdot \mathbb{R}, D$ : $\mathbb{R} \times \mathbb{R} \times D \rightarrow \mathbb{R} \times D$, and the right vertical arrow is $\cdot \mathbb{R}, D: \mathbb{R} \times D \rightarrow D$.

(3)

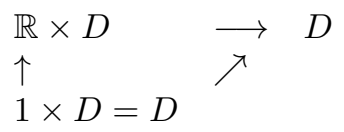

where the horizontal arrow is $\cdot_{\mathbb{R}, D}: \mathbb{R} \times D \rightarrow D$, the vertical arrow is $1_{\mathbb{R}} \times D: 1 \times D \rightarrow \mathbb{R} \times D$, and the slant arrow is $\mathrm{id}_{D}: D \rightarrow D$.

Remark 5.18. We have no canonical addition in $D$. In other words, we could not define addition in $D$ in such a way as

$$
\begin{aligned}
& \mathbf{D}\left((X+Y) /\left(X^{2}, Y^{2}\right) \leftarrow X /\left(X^{2}\right)\right): D \times D=\mathbf{R}[X] /\left(X^{2}\right) \times \mathbf{R}[Y] /\left(Y^{2}\right) \\
= & \mathbf{R}[X, Y] /\left(X^{2}, Y^{2}\right) \rightarrow \mathbf{R}[X] /\left(X^{2}\right)=D .
\end{aligned}
$$

This would simply be meaningless, because

$$
(X+Y) /\left(X^{2}, Y^{2}\right) \leftarrow X /\left(X^{2}\right)
$$

is not well-defined.

Remark 5.19. We have the canonical morphism $D \rightarrow \mathbb{R}$. Specifically speaking, it is to be

$$
\mathbf{D}\left(X /\left(X^{2}\right) \leftarrow Z\right): D=\mathbf{D}\left(\mathbf{R}[X] /\left(X^{2}\right)\right) \rightarrow \mathbf{D}(\mathbf{R}[Z])=\mathbb{R} .
$$

Many significant concepts and theorems of topos theory can quite easily be transferred into the theory of Weil categories, with due modifications of course. In particular, we have

Theorem 5.20. (The Fundamental Theorem for Weil Categories, cf. Theorem 4.19 in [3] and Theorem 1 in $\S I V .7$ of [13]) Let $(\mathcal{K}, \mathbf{D})$ be a Weil category with $M \in \mathcal{K}$. Then the slice category $\mathcal{K} / M$ endowed with a Dubuc functor $\mathbf{D}_{M}: \rightarrow \mathcal{K} / M$ is a Weil category, where

(i) $\mathbf{D}_{M}(A)$ is the canonical projection $\mathbf{D}(A) \times M \rightarrow M$ for any $A \in \widetilde{\mathfrak{W}}$, and 
(ii) $\mathbf{D}_{M}(f)$ is $f \times M$ for any morphism $f$ in $\widetilde{\mathfrak{W}}$.

Remark 5.21. This theorem corresponds to the the so-called fiberwise differential geometry. In other words, the theorem claims that we can do differential geometry fiberwise.

\section{Axiomatic differential geometry}

We fix a Weil category $(\mathcal{K}, \mathbf{D})$ throughout this section.

Notation 6.1. We introduce the following aliases. The entity

$$
\mathbf{D}\left(\mathbf{R}[X, Y] /\left(X^{2}, Y^{2}, X Y\right)\right)
$$

is denoted by $D(2)$ and the entity

$$
\mathbf{D}\left(\mathbf{R}[X, Y, Z] /\left(X^{2}, Y^{2}, Z^{2}, X Y, X Z, Y Z\right)\right)
$$

is denoted by $D(3)$.

As a corollary of Proposition 5.12 and Theorem 5.20, we have

Proposition 6.2. The canonical projection $\mathbb{R} \times M \rightarrow M$ is a commutative $\mathbf{R}$-algebra object in the slice category $\mathcal{K} / M$.

Definition 6.3. An object $M$ in $\mathcal{K}$ is called microlinear provided that a finite limit diagram $\mathcal{D}$ in $\mathfrak{W}$ always yields a limit diagram $\mathbf{T}(\mathcal{D}, M)$ in $\mathcal{K}$.

As in Proposition 4.2, we have

Proposition 6.4. We have the following:

(1) The limit of a diagram of microlinear objects in $\mathcal{K}$ is microlinear.

(2) Given objects $M$ and $N$ in $\mathcal{K}$, if $M$ is microlinear, then the exponential $M^{N}$ is also microlinear.

Theorem 6.5. Let $M$ be a microlinear object in $\mathcal{K}$. The entity

$$
M^{\mathbf{D}\left(\mathbf{R} \rightarrow \mathbf{R}[X] /\left(X^{2}\right)\right)}: M^{D}=M^{\mathbf{D}\left(\mathbf{R}[X] /\left(X^{2}\right)\right)} \rightarrow M^{\mathbf{D}(\mathbf{R}))}=M
$$

is a $(\mathbb{R} \times M \rightarrow M)$-module object in the slice category $\mathcal{K} / M$ with respect to the following addition and scalar multiplication:

(i) The following diagram

$$
\begin{array}{ccc}
\mathbf{R}[X, Y] /\left(X^{2}, Y^{2}, X Y\right) & \rightarrow & \mathbf{R}[Y] /\left(Y^{2}\right) \\
\downarrow & & \downarrow \\
\mathbf{R}[X] /\left(X^{2}\right) & \rightarrow & \mathbf{R}
\end{array}
$$

is a pullback, where the upper horizontal arrow is

$$
(X, Y) /\left(X^{2}, Y^{2}, X Y\right) \rightarrow(0, Y) /\left(Y^{2}\right),
$$

the lower horizontal arrow is

$$
X /\left(X^{2}\right) \rightarrow 0
$$

the left vertical arrow is

$$
(X, Y) /\left(X^{2}, Y^{2}, X Y\right) \rightarrow(X, 0) /\left(X^{2}\right)
$$


and the right vertical arrow is

$$
Y /\left(Y^{2}\right) \rightarrow 0
$$

Since $M$ is microlinear, the diagram

$$
\begin{array}{ccc}
M^{D(2)}=M^{\mathbf{D}\left(\mathbf{R}[X, Y] /\left(X^{2}, Y^{2}, X Y\right)\right)} & \rightarrow & M^{\mathbf{D}\left(\mathbf{R}[Y] /\left(Y^{2}\right)\right)}=M^{D} \\
\downarrow & & \downarrow \\
M^{D}=M^{\mathbf{D}\left(\mathbf{R}[X] /\left(X^{2}\right)\right)} & \rightarrow & M^{\mathbf{D}(\mathbf{R})}=M
\end{array}
$$

is a pullback, where the upper horizontal arrow is

$$
M^{\mathbf{D}\left((X, Y) /\left(X^{2}, Y^{2}, X Y\right) \rightarrow(0, Y) /\left(Y^{2}\right)\right),}
$$

the lower horizontal arrow is

$$
M^{\mathbf{D}\left(X /\left(X^{2}\right) \rightarrow 0\right)}
$$

the left vertical arrow is

$$
M^{\mathbf{D}\left((X, Y) /\left(X^{2}, Y^{2}, X Y\right) \rightarrow(X, 0) /\left(X^{2}\right)\right)}
$$

and the right vertical arrow is

$$
M^{\mathbf{D}\left(Y /\left(Y^{2}\right) \rightarrow 0\right)} .
$$

Therefore, we have

$$
M^{D(2)}=M^{D} \times_{M} M^{D} .
$$

The morphism

$$
\begin{aligned}
& M^{\mathbf{D}\left((X, Y) /\left(X^{2}, Y^{2}, X Y\right) \rightarrow(X, X) /\left(X^{2}\right)\right)}: M^{D} \times_{M} M^{D} \\
= & M^{D(2)}=M^{\mathbf{D}\left(\mathbf{R}[X, Y] /\left(X^{2}, Y^{2}, X Y\right)\right)} \rightarrow M^{\mathbf{D}\left(\mathbf{R}[X] /\left(X^{2}\right)\right)}=M^{D}
\end{aligned}
$$

stands for addition and is denoted by $\varphi$.

(ii) The composition of the morphism

$$
\begin{aligned}
& \mathbf{D}\left(X Y /\left(X^{2}\right) \leftarrow X /\left(X^{2}\right)\right) \times M^{D}: D \times \mathbb{R} \times M^{D} \\
= & \mathbf{D}\left(\mathbf{R}[X] /\left(X^{2}\right)\right) \times \mathbf{D}(\mathbf{R}[Y]) \times M^{D} \rightarrow \mathbf{D}\left(\mathbf{R}[X] /\left(X^{2}\right)\right) \times M^{D}=D \times M^{D}
\end{aligned}
$$

and the evaluation morphism

$$
D \times M^{D} \rightarrow M
$$

is denoted by $\widehat{\psi}_{1}: D \times \mathbb{R} \times M^{D} \rightarrow M$. Its transpose $\psi_{1}: \mathbb{R} \times M^{D} \rightarrow M^{D}$ stands for scalar multiplication.

Proof. Here we deal only with the associativity of addition and the distibutivity of scalar multiplication over addition, leaving verification of the other requisites of $M^{\mathbf{D}\left(\mathbf{R} \rightarrow \mathbf{R}[X] /\left(X^{2}\right)\right)}: M^{D}=M^{\mathbf{D}\left(\mathbf{R}[X] /\left(X^{2}\right)\right)} \rightarrow M^{\mathbf{D}(\mathbf{R}))}=M$ being a $(\mathbb{R} \times M \rightarrow$ $M$ )-module object in the category $\mathcal{K} / M$ to the reader. (i) The diagram

$$
\begin{aligned}
& \mathbf{R}[X, Y, Z] /\left(X^{2}, Y^{2}, Z^{2}, X Y, X Z, Y Z\right)
\end{aligned}
$$

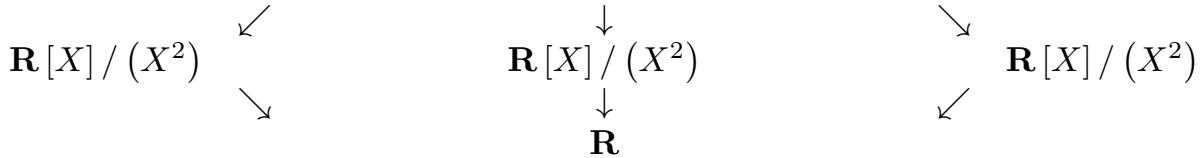


is a limit diagram, where the upper three arrows are

$$
\begin{aligned}
& (X, Y, Z) /\left(X^{2}, Y^{2}, Z^{2}, X Y, X Z, Y Z\right) \rightarrow(X, 0,0) /\left(X^{2}\right), \\
& (X, Y, Z) /\left(X^{2}, Y^{2}, Z^{2}, X Y, X Z, Y Z\right) \rightarrow(0, X, 0) /\left(X^{2}\right), \\
& (X, Y, Z) /\left(X^{2}, Y^{2}, Z^{2}, X Y, X Z, Y Z\right) \rightarrow(0,0, X) /\left(X^{2}\right)
\end{aligned}
$$

from left to right, and the lower three arrows are the same

$$
X /\left(X^{2}\right) \rightarrow 0 \text {. }
$$

Since $M$ is microlinear, the diagram

$$
\begin{aligned}
& M^{D(3)}= \\
& M^{\mathbf{D}\left(\mathbf{R}[X, Y, Z] /\left(X^{2}, Y^{2}, Z^{2}, X Y, X Z, Y Z\right)\right)}
\end{aligned}
$$

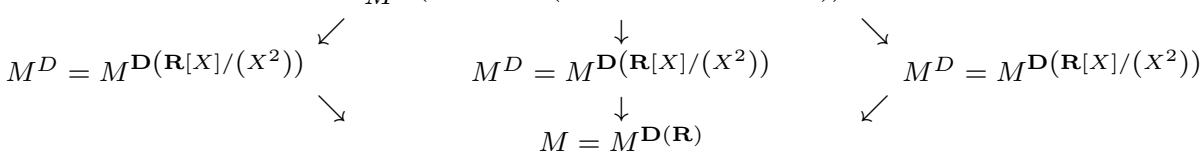

is a limit diagram, where the upper three arrows are

$$
\begin{aligned}
& M^{\mathbf{D}\left((X, Y, Z) /\left(X^{2}, Y^{2}, Z^{2}, X Y, X Z, Y Z\right) \rightarrow(X, 0,0) /\left(X^{2}\right)\right),} \\
& M^{\mathbf{D}\left((X, Y, Z) /\left(X^{2}, Y^{2}, Z^{2}, X Y, X Z, Y Z\right) \rightarrow(0, X, 0) /\left(X^{2}\right)\right),} \\
& M^{\mathbf{D}\left((X, Y, Z) /\left(X^{2}, Y^{2}, Z^{2}, X Y, X Z, Y Z\right) \rightarrow(0,0, X) /\left(X^{2}\right)\right)}
\end{aligned}
$$

from left to right, and the lower three arrows are the same

$$
M^{\mathbf{D}\left(X /\left(X^{2}\right) \rightarrow 0\right)}
$$

Therefore, we have

$$
M^{D(3)}=M^{D} \times_{M} M^{D} \times_{M} M^{D} .
$$

It is now easy to see that the diagram

$$
\begin{array}{ccc}
M^{D} \times_{M} M^{D} \times_{M} M^{D}=M^{D(3)}= & & M^{\mathbf{D}\left(\mathbf{R}[X, Y] /\left(X^{2}, Y^{2}, X Y\right)\right)}=M^{D(2)} \\
M^{\mathbf{D}\left(\mathbf{R}[X, Y] /\left(X^{2}, Y^{2}, Z^{2}, X Y, X Z, Y Z\right)\right)} & \rightarrow & =M^{D} \times_{M} M^{D} \\
\downarrow & & \downarrow \\
M^{D} \times_{M} M^{D}=M^{D(2)} & \rightarrow & M^{\mathbf{D}\left(\mathbf{R}[X] /\left(X^{2}\right)\right)}=M^{D} \\
=M^{\mathbf{D}\left(\mathbf{R}[X, Y] /\left(X^{2}, Y^{2}, X Y\right)\right)} & \rightarrow &
\end{array}
$$

is commutative, where the upper horizontal arrow is

$$
M^{\mathbf{D}\left((X, Y, Z) /\left(X^{2}, Y^{2}, Z^{2}, X Y, X Z, Y Z\right) \rightarrow(X, X, Y) /\left(X^{2}, Y^{2}, X Y\right)\right),}
$$

the lower horizontal arrow is

$$
M^{\mathbf{D}\left((X, Y) /\left(X^{2}, Y^{2}, X Y\right) \rightarrow(X, X) /\left(X^{2}\right)\right),}
$$

the left vertical arrow is

$$
M^{\mathbf{D}\left((X, Y, Z) /\left(X^{2}, Y^{2}, Z^{2}, X Y, X Z, Y Z\right) \rightarrow(X, Y, Y) /\left(X^{2}, Y^{2}, X Y\right)\right)}
$$

and the right vertical arrow is

$$
M^{\mathbf{D}\left((X, Y) /\left(X^{2}, Y^{2}, X Y\right) \rightarrow(X, X) /\left(X^{2}\right)\right)} .
$$

We have just established the associativity of addition. 
(ii) The proof of the distributivity of scalar multiplication over addition is divided into three steps:

(a) The composition of the morphism

$$
\begin{aligned}
& \mathbf{D}\left((X Z, Y Z) /\left(X^{2}, Y^{2}, X Y\right) \leftarrow(X, Y) /\left(X^{2}, Y^{2}, X Y\right)\right) \times M^{D(2)}: \\
& D(2) \times \mathbb{R} \times M^{D(2)} \\
= & \mathbf{D}\left(\mathbf{R}[X, Y] /\left(X^{2}, Y^{2}, X Y\right)\right) \times \mathbf{D}(\mathbf{R}[Z]) \times M^{D(2)} \rightarrow \\
& \mathbf{D}\left(\mathbf{R}[X, Y] /\left(X^{2}, Y^{2}, X Y\right)\right) \times M^{D(2)}=D(2) \times M^{D(2)}
\end{aligned}
$$

and the evaluation morphism

$$
D(2) \times M^{D(2)} \rightarrow M
$$

is denoted by $\widehat{\psi}_{2}: D(2) \times \mathbb{R} \times M^{D(2)} \rightarrow M$. Its transpose is denoted by $\psi_{2}$ : $\mathbb{R} \times M^{D(2)} \rightarrow M^{D(2)}$. The composition of the morphism

$$
\begin{aligned}
& \mathbf{D}\left(\left(X Z_{1}, Y Z_{2}\right) /\left(X^{2}, Y^{2}, X Y\right) \leftarrow(X, Y) /\left(X^{2}, Y^{2}, X Y\right)\right) \times M^{D(2)}: \\
& D(2) \times \mathbb{R} \times \mathbb{R} \times M^{D(2)} \\
= & \mathbf{D}\left(\mathbf{R}[X, Y] /\left(X^{2}, Y^{2}, X Y\right)\right) \times \mathbf{D}\left(\mathbf{R}\left[Z_{1}, Z_{2}\right]\right) \times M^{D(2)} \rightarrow \\
& \mathbf{D}\left(\mathbf{R}[X, Y] /\left(X^{2}, Y^{2}, X Y\right)\right) \times M^{D(2)}=D(2) \times M^{D(2)}
\end{aligned}
$$

and the evaluation morphism

$$
D(2) \times M^{D(2)} \rightarrow M
$$

is denoted by $\hat{\chi}: D(2) \times \mathbb{R} \times \mathbb{R} \times M^{D(2)} \rightarrow M$. Its transpose is denoted by $\chi: \mathbb{R} \times \mathbb{R} \times M^{D(2)} \rightarrow M^{D(2)}$. It is easy to see that the diagram

$$
\begin{aligned}
& \mathbb{R} \times M^{D(2)} \\
& \downarrow \\
& \mathbb{R} \times \mathbb{R} \times M^{D(2)} \stackrel{\searrow}{\longrightarrow} M^{D(2)}
\end{aligned}
$$

commutes, where the vertical arrow is

$$
\begin{aligned}
& \mathbf{D}\left((Z, Z) \leftarrow\left(Z_{1}, Z_{2}\right)\right) \times M^{D(2)}: \mathbb{R} \times M^{D(2)}=\mathbf{D}(\mathbf{R}[Z]) \times M^{D(2)} \rightarrow \\
& \mathbf{D}\left(\mathbf{R}\left[Z_{1}, Z_{2}\right]\right) \times M^{D(2)}=\mathbb{R} \times \mathbb{R} \times M^{D(2)},
\end{aligned}
$$

the horizontal arrow is

$$
\chi: \mathbb{R} \times \mathbb{R} \times M^{D(2)} \rightarrow M^{D(2)}
$$

and the slant arrow is

$$
\psi_{2}: \mathbb{R} \times M^{D(2)} \rightarrow M^{D(2)} .
$$

It is also easy to see that the morphism $\chi: \mathbb{R} \times \mathbb{R} \times M^{D(2)} \rightarrow M^{D(2)}$ can be defined as

$$
\begin{aligned}
& \psi_{2} \times_{M} \psi_{2}: \mathbb{R} \times \mathbb{R} \times M^{D(2)}=\mathbb{R} \times \mathbb{R} \times\left(M^{D} \times_{M} M^{D}\right) \\
= & \left(\mathbb{R} \times M^{D}\right) \times_{M}\left(\mathbb{R} \times M^{D}\right) \rightarrow M^{D} \times_{M} M^{D}=M^{D(2)} .
\end{aligned}
$$


(b) Let us consider the following diagram:

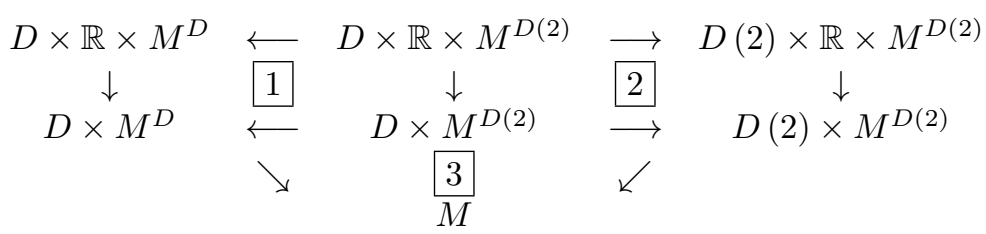

where the upper two horizontal arrows are

$$
\begin{aligned}
& D \times \mathbb{R} \times \varphi: D \times \mathbb{R} \times M^{D(2)} \rightarrow D \times \mathbb{R} \times M^{D} \\
& \mathbf{D}\left((X, X) /\left(X^{2}\right) \leftarrow(X, Y) /\left(X^{2}, Y^{2}, X Y\right)\right) \times \mathbb{R} \times M^{D(2)}: \\
& D \times \mathbb{R} \times M^{D(2)}=\mathbf{D}\left(\mathbf{R}[X] /\left(X^{2}\right)\right) \times \mathbb{R} \times M^{D(2)} \rightarrow \\
& \mathbf{D}\left(\mathbf{R}[X, Y] /\left(X^{2}, Y^{2}, X Y\right)\right) \times \mathbb{R} \times M^{D(2)}=D(2) \times \mathbb{R} \times M^{D(2)}
\end{aligned}
$$

from left to right, the lower two horizontal arrow are

$$
\begin{aligned}
& D \times \varphi: D \times M^{D(2)} \rightarrow D \times M^{D} \\
& \mathbf{D}\left(\begin{array}{c}
(X, X) /\left(X^{2}\right) \leftarrow \\
(X, Y) /\left(X^{2}, Y^{2}, X Y\right)
\end{array}\right) \times M^{D(2)}: D \times M^{D(2)}=\mathbf{D}\left(\mathbf{R}[X] /\left(X^{2}\right)\right) \times M^{D(2)} \\
& \rightarrow \mathbf{D}\left(\mathbf{R}[X, Y] /\left(X^{2}, Y^{2}, X Y\right)\right) \times M^{D(2)}=D(2) \times M^{D(2)}
\end{aligned}
$$

from left to right, the three vertical arrows are

$$
\begin{aligned}
& \mathbf{D}\left(X Y /\left(X^{2}\right) \leftarrow X /\left(X^{2}\right)\right) \times M^{D}: \\
& D \times \mathbb{R} \times M^{D}=\mathbf{D}\left(\mathbf{R}[X] /\left(X^{2}\right)\right) \times \mathbf{D}(\mathbf{R}[Y]) \times M^{D} \\
& \rightarrow \mathbf{D}\left(\mathbf{R}[X] /\left(X^{2}\right)\right) \times M^{D}=D \times M^{D} \\
& \mathbf{D}\left(X Y /\left(X^{2}\right) \leftarrow X /\left(X^{2}\right)\right) \times M^{D(2)}: \\
& D \times \mathbb{R} \times M^{D(2)}=\mathbf{D}\left(\mathbf{R}[X] /\left(X^{2}\right)\right) \times \mathbf{D}(\mathbf{R}[Y]) \times M^{D(2)} \\
& \rightarrow \mathbf{D}\left(\mathbf{R}[X] /\left(X^{2}\right)\right) \times M^{D(2)}=D \times M^{D(2)} \\
& \mathbf{D}\left(\begin{array}{c}
(X Z, Y Z) /\left(X^{2}, Y^{2}, X Y\right) \\
\leftarrow(X, Y) /\left(X^{2}, Y^{2}, X Y\right)
\end{array}\right) \times M^{D(2)}: \\
& D(2) \times \mathbb{R} \times M^{D(2)}=\mathbf{D}\left(\mathbf{R}[X, Y] /\left(X^{2}, Y^{2}, X Y\right)\right) \times \mathbf{D}(\mathbf{R}[Z]) \times M^{D(2)} \rightarrow \\
& \mathbf{D}\left(\mathbf{R}[X, Y] /\left(X^{2}, Y^{2}, X Y\right)\right) \times M^{D(2)}=D(2) \times M^{D(2)}
\end{aligned}
$$

from left to right, and the two slant arrows are the evaluation morphisms $D \times$ $M^{D} \rightarrow M$ and $D(2) \times M^{D(2)} \rightarrow M$. In order to establish the commutativity of the diagram (6.1), we will be engaged in the commutativity of the three subdiagrams 1,2 and 3 in order. It is easy to see that both diagram 1 and diagram 2 commute. The commutativity of diagram 1 is a simple consequence of the fact that $\left(_{-}\right) \times\left({ }_{-}\right)$is a bifunctor, while the commutativity of diagram 2 follows directly from that of the following diagram

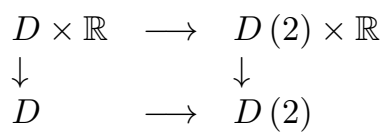


where the two horizontal arrows are

$$
\begin{aligned}
& \mathbf{D}\left((X, X) /\left(X^{2}\right) \leftarrow(X, Y) /\left(X^{2}, Y^{2}, X Y\right)\right) \times \mathbb{R}: \\
& D \times \mathbb{R}=\mathbf{D}\left(\mathbf{R}[X] /\left(X^{2}\right)\right) \times \mathbb{R} \\
& \rightarrow \mathbf{D}\left(\mathbf{R}[X, Y] /\left(X^{2}, Y^{2}, X Y\right)\right) \times \mathbb{R}=D(2) \times \mathbb{R} \\
& \mathbf{D}\left((X, X) /\left(X^{2}\right) \leftarrow(X, Y) /\left(X^{2}, Y^{2}, X Y\right)\right): D=\mathbf{D}\left(\mathbf{R}[X] /\left(X^{2}\right)\right) \rightarrow \\
& \mathbf{D}\left(\mathbf{R}[X, Y] /\left(X^{2}, Y^{2}, X Y\right)\right)=D(2)
\end{aligned}
$$

from top to bottom, and the two vertical arrows are

$$
\begin{aligned}
& \mathbf{D}\left(X Y /\left(X^{2}\right) \leftarrow X /\left(X^{2}\right)\right): \\
& D \times \mathbb{R}=\mathbf{D}\left(\mathbf{R}[X] /\left(X^{2}\right)\right) \times \mathbf{D}(\mathbf{R}[Y]) \\
& \rightarrow \mathbf{D}\left(\mathbf{R}[X] /\left(X^{2}\right)\right)=D \\
& \mathbf{D}\left(\begin{array}{c}
\left.(X Z, Y Z) /\left(X^{2}, Y^{2}, X Y\right) \leftarrow\right) \\
(X, Y) /\left(X^{2}, Y^{2}, X Y\right)
\end{array}\right): \\
& D(2) \times \mathbb{R}=\mathbf{D}\left(\mathbf{R}[X, Y] /\left(X^{2}, Y^{2}, X Y\right)\right) \times \mathbf{D}(\mathbf{R}[Z]) \\
& \rightarrow \mathbf{D}\left(\mathbf{R}[X, Y] /\left(X^{2}, Y^{2}, X Y\right)\right)=D(2)
\end{aligned}
$$

from left to right. The commutativity of diagram 3 follows from the following commutative diagram of the so-called parametrized adjunction (cf. Theorem 3 in $\S I V .7$ of $[12])$ :

$$
\begin{array}{ll}
\operatorname{Hom}_{\mathcal{K}}\left(D(2) \times M^{D(2)}, M\right) & \cong \operatorname{Hom}_{\mathcal{K}}\left(M^{D(2)}, M^{D(2)}\right) \\
\downarrow & \circlearrowleft \downarrow \\
\operatorname{Hom}_{\mathcal{K}}\left(D \times M^{D(2)}, M\right) & \cong \operatorname{Hom}_{\mathcal{K}}\left(M^{D(2)}, M^{D}\right) \\
\uparrow & \circlearrowleft \uparrow \\
\operatorname{Hom}_{\mathcal{K}}\left(D \times M^{D}, M\right) & \cong \operatorname{Hom}_{\mathcal{K}}\left(M^{D}, M^{D}\right)
\end{array}
$$

where the left two vertical arrows are

$$
\begin{gathered}
\operatorname{Hom}_{\mathcal{K}}\left(\left(\begin{array}{c}
\mathbf{D}\left(\begin{array}{c}
(X, X) /\left(X^{2}\right) \leftarrow \\
(X, Y) /\left(X^{2}, Y^{2}, X Y\right)
\end{array}\right): \\
D=\mathbf{D}\left(\mathbf{R}[X] /\left(X^{2}\right)\right) \rightarrow \\
\mathbf{D}\left(\mathbf{R}[X, Y] /\left(X^{2}, Y^{2}, X Y\right)\right)=D(2)
\end{array}\right) \times M^{D(2)}, M\right) \\
\operatorname{Hom}_{\mathcal{K}}\left(D(2) \times M^{D(2)}, M\right) \rightarrow \operatorname{Hom}_{\mathcal{K}}\left(D \times M^{D(2)}, M\right) \\
\operatorname{Hom}_{\mathcal{K}}(D \times \varphi, M): \operatorname{Hom}_{\mathcal{K}}\left(D \times M^{D}, M\right) \rightarrow \operatorname{Hom}_{\mathcal{K}}\left(D \times M^{D(2)}, M\right) .
\end{gathered}
$$

from top to bottom, while the right vertical arrows are

$$
\begin{aligned}
& \operatorname{Hom}_{\mathcal{K}}\left(M^{D(2)}, \varphi\right): \operatorname{Hom}_{\mathcal{K}}\left(M^{D(2)}, M^{D(2)}\right) \rightarrow \operatorname{Hom}_{\mathcal{K}}\left(M^{D(2)}, M^{D}\right) \\
& \operatorname{Hom}_{\mathcal{K}}\left(\varphi, M^{D}\right): \operatorname{Hom}_{\mathcal{K}}\left(M^{D}, M^{D}\right) \rightarrow \operatorname{Hom}_{\mathcal{K}}\left(M^{D(2)}, M^{D}\right)
\end{aligned}
$$

from top to bottom. Choose

$$
\begin{aligned}
\operatorname{id}_{M^{D(2)}} & \in \operatorname{Hom}_{\mathcal{K}}\left(M^{D(2)}, M^{D(2)}\right), \\
\operatorname{id}_{M^{D}} & \in \operatorname{Hom}_{\mathcal{K}}\left(M^{D}, M^{D}\right)
\end{aligned}
$$


on the right-hand side of diagram (6.2). Then, both yield the same morphism in $\operatorname{Hom}_{\mathcal{K}}\left(M^{D(2)}, M^{D}\right)$ if their adjacent vertical arrows are applied. The corresponding morphism of $\operatorname{id}_{M^{D(2)}}$ in $\operatorname{Hom}_{\mathcal{K}}\left(D(2) \times M^{D(2)}, M\right)$ is no other than the evaluation morphism $D(2) \times M^{D(2)} \rightarrow M$, and the corresponding morphism of $\operatorname{id}_{M^{D}}$ in $\operatorname{Hom}_{\mathcal{K}}\left(D \times M^{D}, M\right)$ is no other than the evaluation morphism $D \times M^{D} \rightarrow M$, Therefore, both the evaluation morphisms $D(2) \times M^{D(2)} \rightarrow M$ and $D \times M^{D} \rightarrow M$ yield the same morphism in $\operatorname{Hom}_{\mathcal{K}}\left(D \times M^{D(2)}, M\right)$ by application of their adjacent vertical arrows, which is tantamount to the commutativity of the diagram 3 . We have just established the commutativity of the whole diagram (6.1). In particular, the outer hexagon of diagram (6.1) is commutative, which means that the diagram

$$
\begin{array}{lll}
D \times \mathbb{R} \times M^{D(2)} & \longrightarrow & D(2) \times \mathbb{R} \times M^{D(2)} \\
\downarrow & \circlearrowleft & \downarrow \\
D \times \mathbb{R} \times M^{D} & \longrightarrow & M
\end{array}
$$

is commutative, where the two horizontal arrows are

$$
\begin{aligned}
& \mathbf{D}\left(\begin{array}{c}
(X, X) /\left(X^{2}\right) \leftarrow \\
(X, Y) /\left(X^{2}, Y^{2}, X Y\right)
\end{array}\right) \times \mathbb{R} \times M^{D(2)}: \\
& D \times \mathbb{R} \times M^{D(2)}=\mathbf{D}\left(\mathbf{R}[X] /\left(X^{2}\right)\right) \times \mathbb{R} \times M^{D(2)} \\
& \rightarrow \mathbf{D}\left(\mathbf{R}[X, Y] /\left(X^{2}, Y^{2}, X Y\right)\right) \times \mathbb{R} \times M^{D(2)}=D(2) \times \mathbb{R} \times M^{D(2)} \\
& \widehat{\psi}_{1}: D \times \mathbb{R} \times M^{D} \rightarrow M
\end{aligned}
$$

from top to bottom, and the two vertical arrows are

$$
\begin{gathered}
D \times \mathbb{R} \times \varphi: D \times \mathbb{R} \times M^{D(2)} \rightarrow D \times \mathbb{R} \times M^{D} \\
\widehat{\psi}_{2}: D(2) \times \mathbb{R} \times M^{D(2)} \rightarrow M
\end{gathered}
$$

from left to right.

(c) The following is a commutative diagram of parametrized adjunction (cf. Theorem 3 in $\S$ IV.7 of [12]):

$$
\begin{array}{ll}
\operatorname{Hom}_{\mathcal{K}}\left(D(2) \times \mathbb{R} \times M^{D(2)}, M\right) & \cong \operatorname{Hom}_{\mathcal{K}}\left(\mathbb{R} \times M^{D(2)}, M^{D(2)}\right) \\
\downarrow & \circlearrowleft \downarrow \\
\operatorname{Hom}_{\mathcal{K}}\left(D \times \mathbb{R} \times M^{D(2)}, M\right) & \cong \operatorname{Hom}_{\mathcal{K}}\left(\mathbb{R} \times M^{D(2)}, M^{D}\right) \\
\uparrow & \circlearrowleft \uparrow \\
\operatorname{Hom}_{\mathcal{K}}\left(D \times \mathbb{R} \times M^{D}, M\right) & \cong \operatorname{Hom}_{\mathcal{K}}\left(\mathbb{R} \times M^{D}, M^{D}\right)
\end{array}
$$

where the left two vertical arrows are

$$
\begin{aligned}
& \operatorname{Hom}_{\mathcal{K}}\left(\left(\begin{array}{c}
\mathbf{D}\left(\begin{array}{c}
(X, X) /\left(X^{2}\right) \leftarrow \\
(X, Y) /\left(X^{2}, Y^{2}, X Y\right)
\end{array}\right): \\
D=\mathbf{D}\left(\mathbf{R}[X] /\left(X^{2}\right)\right) \rightarrow \\
\mathbf{D}\left(\mathbf{R}[X, Y] /\left(X^{2}, Y^{2}, X Y\right)\right)=D(2)
\end{array}\right) \times \mathbb{R} \times M^{D(2)}, M\right) \\
& \operatorname{Hom}_{\mathcal{K}}\left(D(2) \times \mathbb{R} \times M^{D(2)}, M\right) \rightarrow \operatorname{Hom}_{\mathcal{K}}\left(D \times \mathbb{R} \times M^{D(2)}, M\right) \\
& \operatorname{Hom}_{\mathcal{K}}(D \times \mathbb{R} \times \varphi, M):
\end{aligned}
$$




$$
\operatorname{Hom}_{\mathcal{K}}\left(D \times \mathbb{R} \times M^{D}, M\right) \rightarrow \operatorname{Hom}_{\mathcal{K}}\left(D \times \mathbb{R} \times M^{D(2)}, M\right)
$$

from top to bottom, while the right vertical arrows are $\operatorname{Hom}_{\mathcal{K}}\left(\mathbb{R} \times M^{D(2)}, \varphi\right): \operatorname{Hom}_{\mathcal{K}}\left(\mathbb{R} \times M^{D(2)}, M^{D(2)}\right) \rightarrow \operatorname{Hom}_{\mathcal{K}}\left(\mathbb{R} \times M^{D(2)}, M^{D}\right)$ $\operatorname{Hom}_{\mathcal{K}}\left(\mathbb{R} \times \varphi, M^{D}\right): \operatorname{Hom}_{\mathcal{K}}\left(\mathbb{R} \times M^{D}, M^{D}\right) \rightarrow \operatorname{Hom}_{\mathcal{K}}\left(\mathbb{R} \times M^{D(2)}, M^{D}\right)$

from top to bottom. Choose

$$
\begin{aligned}
& \widehat{\psi}_{2} \in \operatorname{Hom}_{\mathcal{K}}\left(D(2) \times \mathbb{R} \times M^{D(2)}, M\right) \\
& \widehat{\psi}_{1} \in \operatorname{Hom}_{\mathcal{K}}\left(D \times \mathbb{R} \times M^{D}, M\right)
\end{aligned}
$$

on the left of diagram (6.4). Then both yield the same morphism in $\operatorname{Hom}_{\mathcal{K}}(D \times$ $\left.\mathbb{R} \times M^{D(2)}, M\right)$ if their adjacent vertical arrows are applied by dint of the commutativity of diagram (6.3). The corresponding morphism of $\widehat{\psi}_{2}$ in $\operatorname{Hom}_{\mathcal{K}}(\mathbb{R} \times$ $\left.M^{D(2)}, M^{D(2)}\right)$ is $\psi_{2}$, and the corresponding morphism of $\widehat{\psi}_{1}$ in

$$
\operatorname{Hom}_{\mathcal{K}}\left(\mathbb{R} \times M^{D}, M^{D}\right)
$$

is $\psi_{1}$. Therefore, both $\psi_{2}$ and $\psi_{1}$ yield the same morphism in

$$
\operatorname{Hom}_{\mathcal{K}}\left(\mathbb{R} \times M^{D(2)}, M^{D}\right)
$$

if their adjacent vertical arrows are applied, which is tantamount to the commutativity of the following diagram:

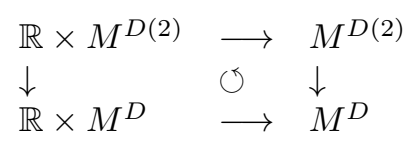

where the two horizontal arrows are

$$
\begin{aligned}
& \psi_{2}: \mathbb{R} \times M^{D(2)} \rightarrow M^{D(2)} \\
& \psi_{1}: \mathbb{R} \times M^{D} \rightarrow M^{D}
\end{aligned}
$$

from top to bottom, and the two vertical arrows are

$$
\begin{gathered}
\mathbb{R} \times \varphi: \mathbb{R} \times M^{D(2)} \rightarrow \mathbb{R} \times M^{D} \\
\varphi: M^{D(2)} \rightarrow M^{D}
\end{gathered}
$$

from left to right. We have just established the distributivity of scalar multiplication over addition.

\section{Concluding Remarks}

Weilology began with André Weil's algebraic treatment of nilpotent infinitesimals [29]. Its second step is synthetic differential geometry [9] and the study of Weil functors of Czech geometers [10]. Its third step is the author's axiomatic differential geometry ([15-22]). Now we have its final form in this paper.

A subsequent paper is devoted to fixing the syntax of Weil categories after the manner of [3], under which we can develop axiomatic differential geometry naively (i.e., without tears), just as René Lavendhomme did for synthetic differential geometry [11]. 
Another important point is that we can investigate Weilology for supergeometry, braided geometry, noncommutative geometry, homotopical differential geometry, arithmetical differential geometry and so on in the same vein, which is the topic of subsequent papers.

\section{REFERENCES}

[1] S. Awodey, Category Theory, 2nd ed., Oxford Logic Guide 52, Oxford University Press, 2010 .

[2] J. C. Baez and A. E. Hoffnung, Convenient categories of smooth spaces, Trans. Am. Math. Soc. 363 (2011), 5789-5825.

[3] J. L. Bell, Toposes and Local Set Theories: an Introduction, Oxford Logic Guide 16, Oxford University Press, 1988.

[4] W. Bertram, Differential Geometry, Lie Groups and Symmetric Spaces over General Base Fields and Rings, Memoirs of the American Mathematical Society 192, American Mathematical Society, 2008.

[5] W. Bertram and A. Souvay, A general construction of Weil functors, Cah. Topol. Géom. Différ. Catég. 55 (2014), 267-313.

[6] W. Bertram, Weil spaces and Weil-Lie groups, 2014, arXiv:1402.2619.

[7] E. J. Dubuc, Sur les modèles de la géométrie différentielle synthétique, Cah. Topol. Géom. Différ. Catég. 20 (1979), 231-279.

[8] P. Iglesias-Zemmour, Diffeology, Mathematical Surveys and Monographs 185, American Mathematical Society, 2013.

[9] A. Kock, Synthetic Differential Geometry, 2nd ed., London Mathematical Society Lecture Note Series 333, Cambridge University Press, 2006.

[10] Kolář, Ivan, Michor, Peter W. and Slovák, Jan, Natrual Operations in Differential Geometry, Springer Verlag 1993.

[11] Lavendhomme, René, Basic Concepts of Synthetic Differential Geometry, Kluwer Texts in the Mathematical Sciences 13, Kluwer Academic Publishers, 1996.

[12] S. Mac Lane, Categories for the Working Mathematician, Graduate Texts in Mathematics 5, Springer-Verlag, 1971.

[13] S. Mac Lane and I. Moerdijk, Sheaves in Geometry and Logic: a First Introduction to Topos Theory, Universitext, Springer-Verlag, 1992.

[14] I. Moerdijk and G. E. Reyes, Models for Smooth Infinitesimal Analysis, Springer-Verlag, 1991.

[15] H. Nishimura, Axiomatic differential geometry I-1 - towards model categories of differential geometry, Math. Appl. 1 (2012), 171-182.

[16] H. Nishimura, Axiomatic differential geometry II-1 - vector fields, Math. Appl. 1 (2012), 183-195.

[17] H. Nishimura, Axiomatic differential geometry II-2 - differential forms, Math. Appl. 2 (2013), 43-60.

[18] H. Nishimura, Axiomatic differential geometry II-3 - its developments - Chapter 3: The general Jacobi identity, Int. J. Pure Appl. Math. 83 (2013), 137-192.

[19] H. Nishimura, Axiomatic differential geometry II-4 - its developments - Chapter 4: the Frölicher-Nijenhuis algebra, Int. J. Pure Appl. Math. 82 (2013), 763-819.

[20] H. Nishimura, Axiomatic differential geometry III-1 - its landscape - Chapter 1: Model theory I, Far East J. Math. Sci. 74 (2013), 17-26.

[21] H. Nishimura, Axiomatic differential geometry III-2 - its landscape - Chapter 2: Model theory II, Far East J. Math. Sci. 74 (2013), 139-154.

[22] H. Nishimura, Axiomatic differential geometry III-3 - its landscape - Chapter 3: The old kingdom of differential geometers, Applied Mathematics 8 (2017), 835-845.

[23] H. Nishimura, A book review of [25], Eur. Math. Soc. Newsl. 99 (2016), 58-60.

[24] H. Nishimura, A review of [25], http://hdl.handle.net/2241/00129866.

[25] F. Paugam, Towards the Mathematics of Quantum Field Theory, Springer, 2014. 
[26] J.-M. Souriau, Groupes differentiels, in: P. L. Garcia, A. Perez-Rendon and J. M. Souriau (eds.), Differential Geometrical Methods in Mathematical Physics, Lecture Notes in Mathematics 836, Springer-Verlag, 1980, 91-128.

[27] A. Stacey, Comparative smootheology, Theory Appl. Categ. 25 (2011), 64-117.

[28] N. Steenrod, A convenient category of topological spaces, Michigan Math. Journal 14 (1967), 133-152.

[29] Weil, André, Théorie des points próches sur les variétés différentiables, in Colloq. Top. et Géom. Diff., Strassbourg, 1953.

Hirokazu Nishimura, Institute of Mathematics, University of Tsukuba, Tsukuba, Ibaraki 3058571, Japan

e-mail: logic@math.tsukuba.ac.jp 
